\title{
Late-Occurring Vancomycin-Associated Acute Kidney Injury in Children Receiving Prolonged Therapy
}

\author{
Chad A. Knoderer, PharmD \\ Allison L. Gritzman \\ Kristen R. Nichols, PharmD \\ Amy C. Wilson, MD
}

\begin{abstract}
:
Background: Acute kidney injury (AKI) in patients receiving vancomycin has been associated with trough concentrations $\geq 15 \mathrm{mg} / \mathrm{L}$ and longer therapy duration. The objective of this study was to determine the incidence and factors associated with late AKI in children receiving $\geq 8$ days of vancomycin therapy.

Methods: Children aged 30 days to 17 years who were admitted to our institution and received intravenous vancomycin for at least 8 days during January to December of 2007 and 2010 and had a suspected or proven gram-positive infection were included. Late AKI was categorized as AKI occurring after the first 7 days of therapy and within 48 hours following vancomycin discontinuation. The primary outcome was incidence of late AKI as determined by modified pRIFLE criteria. Results: One-hundred sixty-seven patients were included, with a median (interquartile range) age (years) and weight (kg) of 2 (1-7) and 12.5 (8.9-23.8). Late AKI was identified in 12.6\% (21/167). A higher percentage of late AKI

patients received concomitant treatment with intravenous acyclovir, amphotericin products, or piperacillin-tazobactam. Age $<1$ year was the only factor independently associated with late AKI development (odds ratio $=4.4 ; 95 \%$ confidence interval $=1.3-15.4$ ). Conclusions: Late AKI occurred in nearly $13 \%$ of children receiving $\geq 8$ days of vancomycin therapy. This study suggests that vancomycin trough concentrations are not associated with late AKI, but that age $<1$ year and concomitant administration of certain nephrotoxins may be factors associated with increased risk.
\end{abstract}

This is the author's manuscript of the article published in final edited form as:

Knoderer, C. A., Gritzman, A. L., Nichols, K. R., \& Wilson, A. C. (2015). Late-occurring vancomycin-associated acute kidney injury in children receiving prolonged therapy. Annals of Pharmacotherapy, 49 (10), 1113-1119. http://dx.doi.org/10.1177/1060028015594190 


\section{Background}

Vancomycin is a glycopeptide antibiotic commonly used to treat gram-positive infections, including those caused by methicillin-resistant Staphylococcus aureus (MRSA). Following consensus recommendations for increased vancomycin dosing, more scrutiny has been directed toward vancomycin-associated acute kidney injury (AKI). ${ }^{1}$ Many studies have been performed to characterize AKI and determine AKI-associated factors in both adult and pediatric cohorts receiving vancomycin. ${ }^{2-11}$ Carreno and colleagues presented a thorough review of the history of vancomycin and nephrotoxicity and provided a comprehensive discussion of studies examining AKI in adult patients. Their review suggested that optimal strategies for safe vancomycin use are still unclear and recommended careful consideration of various risk factors for nephrotoxicity in patients receiving vancomycin. ${ }^{12}$

In children, vancomycin has been traditionally empirically dosed as $40 \mathrm{mg} / \mathrm{kg} / \mathrm{day}$ in 4 divided doses. Pediatric evidence and adult consensus recommendations published in 2009 through 2011 suggested that higher starting doses (eg, $60 \mathrm{mg} / \mathrm{kg} /$ day) may be necessary to achieve serum trough concentrations corresponding to the optimal area under the curve (AUC) for plasma concentration relative to the organism minimum inhibitory concentration (MIC). ${ }^{13-15}$ Subsequently, pediatric reports emerged suggesting increased incidence of AKI. ${ }^{7,8}$ Relative to data in adults, there are fewer pediatric studies evaluating vancomycin-associated AKI, though similar trends have been observed with respect to AKI-associated factors. In some studies, vancomycin-associated AKI in children has been associated with trough concentrations $\geq 15 \mathrm{mg} / \mathrm{L}$ and longer treatment duration. ${ }^{7-}$ 9 A conflicting study performed in critically ill children observed that sustained trough concentrations $\geq 15 \mathrm{mg} / \mathrm{L}$ are not associated with increased AKI. ${ }^{11}$ Many of the pediatric studies adopted an interpretation of the pRIFLE (pediatric risk, injury, failure, loss, and end-stage renal disease) criteria to define AKI by utilizing baseline and postinitiation serum creatinine (SCr) concentration data. ${ }^{7,}$ 9,10,16 None of the currently available pediatric studies specifically addresses the factors associated with late-occurring AKI in patients receiving prolonged courses of vancomycin. The objective of this study was to determine the incidence and factors associated with AKI in children receiving $\geq 8$ days of vancomycin therapy.

\section{Methods}

\section{Study Design and Population}

This was a retrospective, single-center cohort study performed following approval from the institutional review boards at Indiana University and Butler University. Patients aged 30 days through 17 years who received vancomycin for at least 8 days for a suspected or proven grampositive infection were eligible for inclusion. Patients included received vancomycin during the calendar years 2007 and 2010, and these times represented periods before and after the recommended empiric vancomycin dosing was changed from $40 \mathrm{mg} / \mathrm{kg} / \mathrm{day}$ to $60 \mathrm{mg} / \mathrm{kg} / \mathrm{day}$ within our institution. It is important to note that despite these dose recommendations, empiric doses during both time periods ultimately remained at the discretion of individual prescribers in the course of routine practice. 
Our goal in this study was to characterize late-occurring vancomycin-associated AKI. Since included patients received vancomycin for at least 8 days, we stratified patients into 2 groups. We defined early AKI as any AKI that occurred within the first 7 days of vancomycin therapy. Late AKI was defined as AKI that occurred at any time from day 8 and beyond of vancomycin therapy. We then excluded patients characterized as having early AKI to specifically examine the patients who experienced AKI later in the course of their vancomycin therapy- "late AKI" patients. Exclusions were made for patients with baseline SCr above institutional age-adjusted normal values (Table 1) prior to vancomycin initiation and for patients with either no baseline or no follow-up SCr obtained during the vancomycin course. Receipt of dialysis or extracorporeal membrane oxygenation was not a specific exclusion criterion. Baseline SCr was defined as a SCr obtained within 48 hours prior to initiating vancomycin therapy. Patients with an underlying diagnosis of cystic fibrosis were also excluded due to their altered pharmacokinetic properties of other antibiotics and potential need for higher vancomycin doses. In the instance where a patient received multiple vancomycin courses during the same hospitalization, only data from the first vancomycin course was recorded.

Baseline demographic and clinical data, vancomycin dosing information, and history of exposure to other factors known to be associated with risk of AKI (ie, intensive care unit admission, extracorporeal membrane oxygenation utilization, sepsis) were extracted from medical records of eligible patients. Coding information from the institution's information management system was used to identify patients with sepsis. Receipt of concurrent nephrotoxins, including aminoglycosides, tacrolimus, meropenem, piperacillin-tazobactam, cyclosporine, scheduled NSAIDs (nonsteroidal anti-inflammatory drugs), intravenous (IV) acyclovir, amphotericin B deoxycholate, and IV iodinated contrast, was recorded. Steady-state vancomycin serum trough concentrations and SCr concentrations were recorded during vancomycin administration and for 10 days following vancomycin discontinuation. Though an optimal target range for vancomycin trough concentrations has not been identified in children, 10 to $15 \mathrm{mg} / \mathrm{L}$ was chosen for the purpose of this study. Patients' initial vancomycin trough serum concentrations were categorized as above $(\geq 15 \mathrm{mg} / \mathrm{L})$ or within/below $(<15 \mathrm{mg} / \mathrm{L})$ target range.

\section{Outcomes}

Acute kidney injury was defined as an increase in SCr by $\geq 50 \%$ from baseline, as adapted from pRIFLE criteria. ${ }^{16} \mathrm{SCr}$ was used as a surrogate marker for estimated creatinine clearance to minimize calculation steps as these values are inversely proportional. ${ }^{16}$ Early AKI was categorized as occurring within the first 7 days of vancomycin therapy. Late AKI was categorized as AKI occurring after the first 7 days of treatment or up to 48 hours following vancomycin discontinuation. The primary outcome analyzed was late AKI incidence as determined by pRIFLE criteria.

\section{Data Analysis}

Baseline demographics and clinical characteristics were compared between groups with and without late AKI using independent-samples $t$ tests, $\chi^{2}$ analyses, and Mann-Whitney tests for nonparametric data. Continuous data were described using mean and standard deviation (SD) for 
variables considered to be normally distributed and median and interquartile range (IQR) for variables considered to be nonnormally distributed. To determine factors associated with late AKI, a logistic regression model was utilized using a forced entry method. The regression model included variables considered to be clinically significant or having a $\mathrm{P}$ value $<0.25$ after univariate analysis. $\mathrm{P}$ values of less than 0.05 were considered to be statistically significant. Statistical analyses were conducted using Statistical Package for Social Sciences version 19.0 (SPSS, Inc, Chicago, IL).

\section{Results}

Two hundred fifty-one $(\mathrm{N}=251)$ patients received vancomycin for 8 days or longer and were included. Early AKI was identified in 19.9\% (50/251); those patients were excluded from further study. Of the remaining patients without early AKI, follow-up SCr data were unavailable in 16.9\% (34/201), leaving 167 patients for final analysis (Figure 1). Patients in the final analysis had a median (IQR) age (years) of 2 (1-7) with a minimum to maximum age of 1 month to 17 years. Median age did not differ between groups (Table 2). However, there were a higher percentage of patients less than 1 year of age in the late AKI group (38.1\% vs $18.5 \%, \mathrm{P}=0.039$ ). Patients in the final analysis had a median (IQR) weight (kg) of 12.5 (8.9-23.8). The mean \pm SD duration of vancomycin treatment was $13.2 \pm 5.5$ days. Late AKI was identified in $12.6 \%(21 / 167)$ of these children. On evaluation of the total incidence of AKI, the early AKI patients were included in the analysis, and it was found that 71 of 217 evaluable patients (32.7\%) experienced AKI (early or late) during their vancomycin treatment course. Table 2 summarizes univariate analysis and clinical characteristics of those with no AKI and with late AKI.

Mean duration of vancomycin treatment was similar between patients with and without AKI, but a significantly higher proportion of patients with AKI received vancomycin courses exceeding 2 weeks duration; $52.4 \%$ of patients with late AKI received $\geq 15$ days treatment versus $30.1 \%$ of patients with no AKI ( $\mathrm{P}=0.043$; observed post hoc power 0.53$)$. There was no difference in initial serum trough concentrations between those with and without late AKI (Table 2). Both the no AKI and late AKI groups were similar in the percentage of patients maintaining a trough concentration $\geq 15 \mathrm{mg} / \mathrm{L}(10.4 \%$ vs $5.3 \%, \mathrm{P}=0.695)$. The maximum trough concentration $(\mathrm{mg} / \mathrm{L})$ in the no AKI group (median [IQR]: 9.7 [7.1-12.8]) was not different than the maximum vancomycin trough concentration prior to AKI in the late AKI group (median [IQR]: 10.2 [8.9-14.1], P = 0.264). The highest reported trough concentrations for the no AKI and late AKI groups were 45.7 and 18.9 $\mathrm{mg} / \mathrm{L}$, respectively.

Sepsis or oncologic diagnoses were more common in children with late AKI. Additionally, a higher percentage of patients with late AKI received concomitant treatment with IV acyclovir, amphotericin products, or piperacillin-tazobactam (Table 1). Piperacillin-tazobactam in combination with either an amphotericin product $(2.1 \%$ vs $14.3 \%, \mathrm{P}=0.027$ ) or IV acyclovir (3.4\% vs $19 \%, \mathrm{P}=0.016$ ) was also encountered with higher frequency in late AKI patients compared to those without late AKI. Late AKI patients also received the combination of piperacillin-tazobactam plus aminoglycoside more frequently than those patients without AKI, though this difference did not reach statistical significance (13\% vs $28.6 \%, \mathrm{P}=0.094)$. 
The following variables met criteria as candidate variables to be included in the multivariate logistic regression model: age $<1$ year, maximum trough concentration, vancomycin duration $\geq 15$ days, oncologic diagnosis, sepsis, receipt of any concurrent nephrotoxin, acyclovir, amphotericin, aminoglycoside, and piperacillin-tazobactam. Following multivariate analysis, age $<1$ year was the only factor independently associated with late AKI development (odds ratio $=4.4 ; 95 \%$ confidence interval = 1.3-15.4; Table 3).

\section{Discussion}

Vancomycin utilization and dosing to achieve optimal efficacy and safety has undergone increased scrutiny within the past decade. Recommendations for increased empiric vancomycin dosing in adult patients resulted from an observation of decreasing susceptibility of methicillin-resistant Staphylococcus aureus to vancomycin and consideration of vancomycin's pharmacodynamics parameters. ${ }^{1}$ In adults, an increased vancomycin dose may be more likely to achieve the goal AUC-MIC ratio, which is the main predictor of vancomycin efficacy. ${ }^{1}$ The AUC-MIC ratio cannot be efficiently determined in practice, and the serum trough concentration is recommended as an appropriate surrogate with a goal value of 15 to $20 \mathrm{mg} / \mathrm{L}$ to correspond with an AUC-MIC ratio at least 400:1. ${ }^{1}$ Pediatric data published after the consensus recommendations bring into question the correlation of trough concentration to AUC in children. ${ }^{17,18}$ Studies by Le et al ${ }^{17}$ and Frymoyer et $\mathrm{al}^{18}$ suggest that in children, a vancomycin trough concentration of 7 to $10 \mathrm{mg} / \mathrm{L}$ is more closely correlated with an AUC-MIC ratio of 400:1. Targeting higher trough concentrations of 15 to $20 \mathrm{mg} / \mathrm{L}$, which would lead to higher AUC-MIC values, could increase the potential for vancomycin-associated nephrotoxicity. ${ }^{17,18}$ Optimal strategies for monitoring vancomycin in children remain controversial. ${ }^{19}$

Vancomycin has traditionally been viewed as a nephrotoxic agent, and recent reviews are available discussing potential explanations for the physiologic basis of vancomycin-associated nephrotoxicity, including continued presence of fermentation by-products, direct oxidative stress, or allergic interstitial nephritis. ${ }^{12,20}$ Adult patients experiencing initial vancomycin serum trough concentrations $\geq 15 \mathrm{mg} / \mathrm{L}$ or receiving therapy durations $\geq 7$ days may be at higher AKI risk. ${ }^{2 \Downarrow \Downarrow \Downarrow-6}$ McKamy and colleagues suggested that children with high vancomycin serum trough concentrations ( $\geq 15 \mathrm{mg} / \mathrm{L}$ ) were nearly 3 times more likely to have nephrotoxicity compared to children with low trough concentrations. ${ }^{8}$ We have previously published data which suggest that an initial trough concentration $\geq 15 \mathrm{mg} / \mathrm{L}$ is a predictor of AKI within the first 7 days of vancomycin therapy. ${ }^{7}$ Bias is a risk in both of these studies as patients with undetected renal dysfunction would be expected to have higher vancomycin levels. Vancomycin dose, duration, ICU admission, and concurrent nephrotoxin receipt have all been suggested, through logistic regression models, to be predictors of AKI in children. ${ }^{7-11}$ Cies and Shankar suggested that while vancomycin duration may be associated with nephrotoxicity, trough concentrations $\geq 15 \mathrm{mg} / \mathrm{L}$ were not. ${ }^{11}$ Unfortunately, differences in study size, design, and cohort characteristics, as well as the retrospective nature of all of these studies, have likely contributed to inconsistent findings as it pertains to determinants of the true risk factors for vancomycin-associated AKI.

In our cohort of patients, the late AKI incidence was nearly $13 \%$ in patients who received vancomycin for at least 8 days (without AKI during the first week of therapy); this incidence is 
consistent with previous reports, in which rates of late AKI range from $13.7 \%$ to $19.4 \%{ }^{6 \Downarrow \Downarrow \Downarrow-10}$ Though our univariate analysis of clinical factors that may associate with late vancomycin-related AKI risk demonstrated findings consistent with other prior studies, our study did not observe those same associations in multivariate analysis. Rather, our multivariate analysis suggests that age $<1$ year was the only factor independently associated with late AKI. Patients less than 1 year of age and receiving vancomycin $\geq 8$ days have a 4.4 times higher odds of developing late AKI than patients greater than 1 year. It is possible that some of the factors, such as trough concentration, dose, or concurrent administration of additional nephrotoxins, are truly more impactful earlier in therapy and perhaps AKI risk evolves over the duration of the vancomycin course. Vancomycin duration appears to be a common factor associated with AKI, but a limitation of prior pediatric studies is the lack of specific characterization of late AKI and factors associated with it. Studies by Cies and Shankar ${ }^{11}$ and Sinclair et $\mathrm{al}^{9}$ suggest that vancomycin duration is an independent predictor of AKI, but neither study identified a particular time point at which AKI risk increases. In our cohort of patients receiving at least 8 days of vancomycin therapy, those receiving $\geq 15$ days were 2.6 times $(95 \%$ confidence interval $=1.01-6.44)$ more likely to experience late AKI than those receiving a more modest 8- to 14-day course.

We found several clinically important associations that are noteworthy. Vancomycin serum trough concentrations were not associated with late AKI in the univariate analyses, which could mean that other factors beyond vancomycin concentrations are more important in the development of late AKI. Significantly fewer children in the late AKI group received NSAIDs. We suspect that this finding may be because the most severely ill children (ie, sepsis, oncologic diagnoses, etc), those who are highly susceptible to toxic vancomycin effects, are generally not treated with NSAIDs. Receipt of concurrent acyclovir, aminoglycosides, amphotericin, or piperacillintazobactam did not reach statistical significance in the multivariate model, but significant associations were found following univariate analysis and these should remain clinically important considerations for practitioners. In particular, results from recent adult studies have suggested that concurrent piperacillin-tazobactam may increase risk for vancomycin-associated AKI. ${ }^{21,22}$ Significantly more patients with late AKI in our cohort received combination therapy with piperacillin-tazobactam (61.9\% vs 38.4\%; Table 1). Additionally, our findings suggest that piperacillin-tazobactam in combination with either an amphotericin product or IV acyclovir may be an important factor in the development of late AKI. Nearly $20 \%$ of our patients with an oncologic diagnosis were receiving an amphotericin product, over half were receiving piperacillintazobactam, and nearly $10 \%$ were receiving both drugs in addition to vancomycin. This observation increased the difficult in truly determining if the concurrent receipt of these products or the underlying oncologic disease is the true risk factor for AKI. Meropenem was likely a marker of severity in our population, but we included it in our analysis as an additional beta-lactam antibiotic for comparison to piperacillin-tazobactam. ${ }^{23}$ The clinical challenge that remains is how to most optimally consider these findings along with previous reports to continuously assess an ever-changing pediatric patient's vancomycin-associated AKI risk.

There are limitations to this study including its retrospective design and the relatively small sample size, which may have limited our availability to identify true risk factors for vancomycinassociated AKI. Our study likely lacked the necessary power due to the small sample size or 
retrospective design. The inherent limitations of reliance on chart documentation and the variable availability of certain data could have affected our findings, as well as the generalizability of our findings. Our study, as is common to several of the previous pediatric studies, utilized pRIFLE criteria and SCr data to define AKI. ${ }^{7,9,10,16}$ A drawback of using this is relying on SCr data, which may not be reflective of a patient's true renal function. Transient or small normal variations in a child's SCr, including variance due to acceptable laboratory error, could arguably place the child into a pRIFLE category of AKI without clinical evidence supporting nephrotoxicity. Patients in this cohort with very low baseline $\mathrm{SCr}(0.2-0.3 \mathrm{mg} / \mathrm{dL})$ could have been categorized as experiencing AKI when it is possible that they experienced an "increase” in SCr due to laboratory error or normal variation as opposed to true impairment in glomerular filtration. Though we recognize its limitations, we would, however, argue that the merits of the pRIFLE classification system as a standardized tool used to monitor for pediatric AKI make it the most robust method for AKI identification available for use in a retrospective study such as this. Two different laboratory methods for determining SCr values were utilized in our institution during the study period. In December of 2008 our institution switched from using enzymatic creatinine assays to the isotrope dilution mass spectroscopy traceable Jaffe method. Because incidence of AKI was determined based on the percent change from baseline SCr values, we would not suspect this to be a significant impairment to our study.

\section{Conclusions}

In this retrospective cohort study of 167 children who received $\geq 8$ days of vancomycin therapy, late AKI occurred in nearly $13 \%$ of patients. Age $<1$ year, vancomycin duration $\geq 15$ days, presence of sepsis, presence of oncologic diagnoses, and concomitant administration of IV acyclovir, amphotericin, or piperacillin-tazobactam were all factors found to be associated with an increased risk of late AKI. Vancomycin trough concentrations were not found to be associated with late AKI. Our study highlights the need for improved prospective study of vancomycin-associated AKI risk assessment tools.

\section{Article Notes}

Authors' Note Presented in part at the 54th Interscience Conference on Antimicrobial Agents and Chemotherapy; September 5-9, 2014; Washington, DC.

Declaration of Conflicting Interests The author(s) declared no potential conflicts of interest with respect to the research, authorship, and/or publication of this article.

Funding The author(s) disclosed receipt of the following financial support for the research, authorship, and/or publication of this article: Chad A. Knoderer and Allison L. Gritzman received financial support for the research, in part, through the Butler University Summer Institute.

\section{References}

1. Rybak MJ, Lomaestro B, Rotschafer JC, et al. Therapeutic monitoring of vancomycin in adult patients: a consensus review of the American Society of Health-System Pharmacists, the Infectious Diseases Society of America, and the Society of Infectious Diseases Pharmacists. Am J Health Syst Pharm. 2009;66:82-98.

2. Cano EL, Haque NZ, Welch VL, et al. Incidence of nephrotoxicity and association with vancomycin use in intensive care unit patients with pneumonia: retrospective analysis of the IMPACT-HAP database. Clin Ther. 2012;34:149-157. 
3. Hidayat LK, Hsu DI, Quist R, Shriner KA, Wong-Beringer A. High-dose vancomycin therapy for methicillinresistant Staphylococcus aureus infections. Arch Intern Med. 2006;166:2138-2144.

4. Pritchard L, Baker C, Legget J, Sehdev P, Brown A, Bayley KB. Increasing vancomycin serum trough concentrations and incidence of nephrotoxicity. Am J Med. 2010;123:1143-1149.

5. Bosso JA, Nappi J, Rudisill C, et al. Relationship between vancomycin trough concentrations and nephrotoxicity: a prospective multicenter trial. Antimicrob Agents Chemother. 2011;55:5475-5479.

6. Jeffres MN, Isakow W, Doherty JA, Micek ST, Kollef MH. A retrospective analysis of possible renal toxicity associated with vancomycin in patients with health care-associated methicillin-resistant Staphylococcus aureus pneumonia. Clin Ther. 2007;29:1107-1115.

7. Knoderer CA, Nichols KR, Lyon KC, Veverka MM, Wilson AC. Are elevated vancomycin serum trough concentrations achieved within the first 7 days of therapy associated with acute kidney injury in children [published online November 11, 2013]? J Pediatr Infect Dis. doi:10.1093/jpids/pit076.

8. McKamy S, Hernandez E, Jahng M, Moriwaki T, Deveikis A, Le J. Incidence and risk factors influencing the development of vancomycin nephrotoxicity in children. J Pediatr. 2011;158:422-426.

9. Sinclair EA, Yenokyan G, McMunn A, Fadrowski JJ, Milstone AM, Lee CK. Factors associated with acute kidney injury in children receiving vancomycin. Ann Pharmacother. 2014;48:1555-1562.

10. Totapally BR, Machado J, Lee H, Paredes A, Rasynski A. Acute kidney injury during vancomycin therapy in critically ill children. Pharmacotherapy. 2013;33:598-602.

11. Cies JJ, Shankar V. Nephrotoxicity in patients with vancomycin trough concentrations of $15-20 \mu \mathrm{g} / \mathrm{ml}$ in a pediatric intensive care unit. Pharmacotherapy. 2013;33:392-400.

12. Carreno JJ, Kenney RM, Lomaestro B. Vancomycin-associated renal dysfunction: where are we now? Pharmacotherapy. 2014;34:1259-1268.

13. Frymoyer A, Hersh AL, Benet LZ, Guglielmo BJ. Current recommended dosing of vancomycin for children with invasive methicillin-resistant Staphylococcus aureus infections is inadequate. Pediatr Infect Dis J. 2009;28:398-402.

14. Frymoyer A, Guglielmo BJ, Wilson SD, Scarpace SB, Benet LZ, Hersh AL. Impact of a hospital wide increase in empiric pediatric vancomycin dosing on initial trough concentrations. Pharmacotherapy. 2011;31:871-876.

15. Eiland LS, English TM, Eiland EH. Assessment of vancomycindosing and subsequent serum concentrations in pediatric patients. Ann Pharmacother. 2011;45:582-589.

16. Akcan-Arikan A, Zappitelli M, Loftis LL, Washburn KK, Jefferson LS, Goldstein SL. Modified RIFLE criteria in critically ill children with acute kidney injury. Kidney Int. 2007;71:1028-1035.

17. Le J, Bradley JS, Murray W, et al. Improved vancomycin dosing in children using area under the curve exposure. Pediatr Infect Dis J. 2013;32:e155-e163.

18. Frymoyer A, Guglielmo BJ, Hersh AL. Desired vancomycin trough serum concentration for treating invasive methicillin-resistant staphylococcal infections. Pediatr Infect Dis J. 2013;32:1077-1079. doi:10.1097/INF.0b013e318299f75c.

19. Le J, Ngu B, Bradley JS, et al. Vancomycin monitoring in children using Bayesian estimation. Ther Drug Monit. 2014;36:510-518.

20. Patzer L. Nephrotoxicity as a cause of acute kidney injury in children. Pediatr Nephrol. 2008;23:2159-2173.

21. Burgess LD, Drew RH. Comparison of the incidence of vancomycin-induced nephrotoxicity in hospitalized patients with and without concomitant piperacillin-tazobactam. Pharmacotherapy. 2014;34:670-676.

22. Gomes DM, Smotherman C, Birch A, et al. Comparison of acute kidney injury during treatment with vancomycin in combination with piperacillin-tazobactam or cefepime. Pharmacotherapy. 2014;34:662-669.

23. Fisher BT, Zaoutis TE, Leckerman KH, Localio R, Aplenc R. Risk factors for renal failure in pediatric patients with acute myeloid leukemia: a retrospective cohort study. Pediatr Blood Cancer. 2010;55:655-661. 
Table I. Institutional Age-Adjusted Normal Serum Creatinine Values.

\begin{tabular}{lccc}
\hline Age & Male & Age & Female \\
\hline $0-1$ year & $0.2-0.6 \mathrm{mg} / \mathrm{dL}$ & $0-1$ year & $0.3-0.5 \mathrm{mg} / \mathrm{dL}$ \\
$1-5$ years & $0.2-0.7 \mathrm{mg} / \mathrm{dL}$ & $1-3$ years & $0.3-0.6 \mathrm{mg} / \mathrm{dL}$ \\
$5-6$ years & $0.3-0.8 \mathrm{mg} / \mathrm{dL}$ & $3-5$ years & $0.2-0.7 \mathrm{mg} / \mathrm{dL}$ \\
$6-9$ years & $0.3-0.9 \mathrm{mg} / \mathrm{dL}$ & $5-6$ years & $0.3-0.7 \mathrm{mg} / \mathrm{dL}$ \\
$9-10$ years & $0.2-1.1 \mathrm{mg} / \mathrm{dL}$ & $6-10$ years & $0.3-0.8 \mathrm{mg} / \mathrm{dL}$ \\
$10-12$ years & $0.3-0.9 \mathrm{mg} / \mathrm{dL}$ & $10-13$ years & $0.3-0.9 \mathrm{mg} / \mathrm{dL}$ \\
$12-13$ years & $0.3-1.1 \mathrm{mg} / \mathrm{dL}$ & $13-14$ years & $0.4-0.9 \mathrm{mg} / \mathrm{dL}$ \\
$13-14$ years & $0.2-1.2 \mathrm{mg} / \mathrm{dL}$ & $14-15$ years & $0.2-1.1 \mathrm{mg} / \mathrm{dL}$ \\
$14-16$ years & $0.3-1.2 \mathrm{mg} / \mathrm{dL}$ & $15-16$ years & $0.4-1.0 \mathrm{mg} / \mathrm{dL}$ \\
$16-18$ years & $0.4-1.2 \mathrm{mg} / \mathrm{dL}$ & $16-18$ years & $0.3-1.1 \mathrm{mg} / \mathrm{dL}$ \\
$18-150$ years & $0.8-1.4 \mathrm{mg} / \mathrm{dL}$ & $18-150$ years & $0.5-1.2 \mathrm{mg} / \mathrm{dL}$ \\
\hline
\end{tabular}

Table 2. Clinical Characteristics of Patients With No AKI and With Late AKI.

\begin{tabular}{|c|c|c|c|}
\hline & No AKI (I46 of I67; 87.4\%) & Late AKI (2I of I67; 12.6\%) & $P$ Value \\
\hline \multicolumn{4}{|l|}{ Patient characteristics } \\
\hline Male $^{a}$ & $81(55.5)$ & $13(61.9)$ & 0.579 \\
\hline Age (years) ${ }^{b}$ & $2(1-7)$ & I (0.08-5.5) & 0.22 \\
\hline Age less than I year ${ }^{a}$ & $27(18.5)$ & $8(38.1)$ & 0.039 \\
\hline Initial SCr $(\mathrm{mg} / \mathrm{dL})^{\mathrm{c}}$ & $0.45(0.22)$ & $0.37(0.16)$ & 0.106 \\
\hline \multicolumn{4}{|l|}{ Vancomycin information } \\
\hline Empiric vancomycin dose $(\mathrm{mg} / \mathrm{kg} / \text { day })^{\mathrm{c}}$ & $41.2(16.6)$ & $47.5(14.6)$ & 0.102 \\
\hline Empiric vancomycin dose $\geq 60 \mathrm{mg} / \mathrm{kg} /$ day $^{\mathrm{a}}$ & $38(26)$ & $7(33.3)$ & 0.48 \\
\hline Vancomycin duration (days) ${ }^{c}$ & $13.1(5.5)$ & $14.9(5.3)$ & 0.164 \\
\hline Vancomycin duration $\geq 15$ days ${ }^{2}$ & $44(30.1)$ & II (52.4) & 0.043 \\
\hline Initial median vancomycin serum trough concentration $(\mathrm{mg} / \mathrm{L})^{\mathrm{b}}$ & $7.5(5.8-10.4)$ & $7.6(5.9-12.6)$ & 0.751 \\
\hline Initial vancomycin serum trough concentration $\geq 15 \mathrm{mg} / \mathrm{L}^{\mathrm{a}, \mathrm{d}}$ & $10(8.2)$ & $2(11.1)$ & 0.653 \\
\hline \multicolumn{4}{|l|}{ Nephrotoxic factors ${ }^{\mathrm{a}}$} \\
\hline Dehydration & $6(4.1)$ & $2(9.5)$ & 0.265 \\
\hline ECMO & $8(5.5)$ & I (4.8) & NS \\
\hline ICU admission & $59(40.4)$ & $9(42.9)$ & 0.831 \\
\hline Oncologic diagnosis & $44(30.1)$ & $11(52.4)$ & 0.043 \\
\hline Sepsis & $28(19.2)$ & $9(42.9)$ & 0.023 \\
\hline \multicolumn{4}{|l|}{ Nephrotoxic medications ${ }^{\mathrm{a}}$} \\
\hline Any concomitant nephrotoxic medication & $102(69.9)$ & $18(85.7)$ & 0.131 \\
\hline Acyclovir (IV) & $17(11.6)$ & $6(28.6)$ & 0.046 \\
\hline Amphotericin & $10(6.8)$ & $4(19)$ & 0.08 \\
\hline Aminoglycoside & $54(37)$ & II (52.4) & 0.176 \\
\hline Calcineurin inhibitor & $16(11)$ & $3(14.3)$ & 0.712 \\
\hline Contrast dye & $78(53.4)$ & $13(61.9)$ & 0.466 \\
\hline Meropenem & $30(20.5)$ & $6(28.6)$ & 0.402 \\
\hline NSAID & $49(33.6)$ & $2(9.5)$ & 0.024 \\
\hline Piperacillin-tazobactam & $56(38.4)$ & $13(61.9)$ & 0.04 \\
\hline
\end{tabular}

Abbreviations: AKI, acute kidney injury; $\mathrm{SCr}$, serum creatinine; ICU, intensive care unit; ECMO, extracorporeal membrane oxygenation; NS, nonsignificant; IV, intravenous; NSAID, nonsteroidal anti-inflammatory drug.

2Data reported as $n(\%)$.

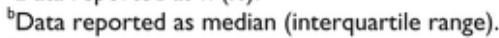

'Data reported as mean (standard deviation).

${ }^{\mathrm{D}}$ Percentages based on patients in each group with a trough concentration obtained ( $n=122$ for no AKI and $n=18$ for late AKI). 
Table 3. Results From Logistic Regression Analysis of Late AKI.

\begin{tabular}{llll}
\hline & OR & $95 \% \mathrm{Cl}$ & $P$ Value \\
\hline Candidate variables & & & \\
Age $<1$ year & 4.4 & $1.3-15.4$ & 0.02 \\
Vancomycin duration $\geq 15$ days & 2.43 & $0.82-7.2$ & 0.11 \\
Oncologic diagnosis & 2.31 & $0.57-9.3$ & 0.241 \\
Sepsis & 2.45 & $0.72-8.3$ & 0.152 \\
Any concomitant nephrotoxic medication & 2.89 & $0.51-16.5$ & 0.232 \\
Acyclovir (IV) & 1.89 & $0.31-11.5$ & 0.49 \\
Amphotericin & 1.61 & $0.33-7.9$ & 0.556 \\
Aminoglycoside & 0.465 & $0.11-2.0$ & 0.303 \\
Piperacillin-tazobactam & 2.77 & $0.97-7.9$ & 0.058 \\
Maximum vancomycin trough concentration prior to AKI & 0.98 & $0.87-1.1$ & 0.671 \\
\hline
\end{tabular}

Abbreviations: $\mathrm{AKI}$, acute kidney injury; $\mathrm{Cl}$, confidence interval; OR, odds ratio; IV, intravenous.

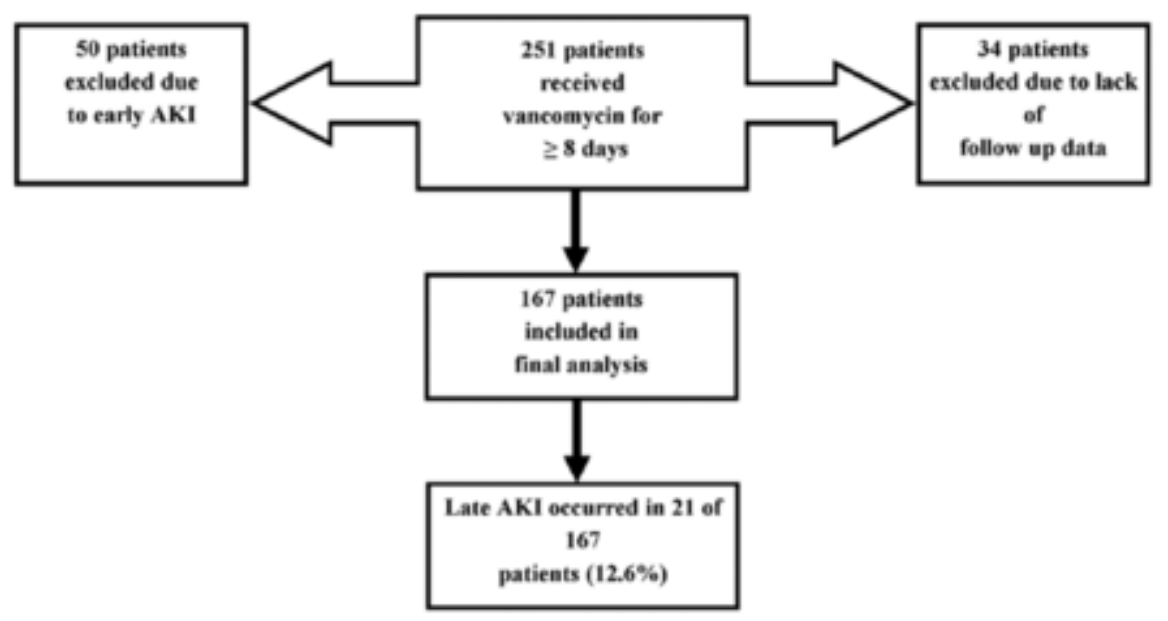

Figure 1 Included patients. 